\title{
FAST CLASSICAL AND QUANTUM FRACTIONAL HAAR WAVELET TRANSFORMS
}

\author{
Valeri Labunets, Ekaterina Labunets-Rundblad, Jaakko Astola \\ Tampere University of Technology, \\ Tampere International Center for Signal Processing \\ Tampere, Finland \\ lab@cs.tut.fi, lab@cs.tut.fi,jta@cs.tut.fi
}

\begin{abstract}
The fractional Fourier transform (FRFT) is oneparametric generalization of the classical Fourier transform. FRFT was introduced in eighties and found a lot of applications in signal processing. The time and spectral domains are both the special cases of the fractional Fourier domain. They correspond to the Oth and Ist fractional Fourier domains, respectively. In this paper, we introduce the classical and quantum fractional Haar-Wavelet transforms and develop corresponding fast algorithms.
\end{abstract}

\section{Introduction}

The singular-value decomposition (SVD) and eigendecomposition (ED) is a tool of both practical and theoretical importance in digital signal processing. The SVD an ED transforms are applicable to many image processing problems such as image coding and restoration, data compression, and power spectrum analysis. They are defined following way.

Let $\mathcal{M}=\left[M_{k}(i)\right]_{\omega, t=0}^{N-1}$ be an arbitrary discrete nonsingular $(N \times N)$-transform. We form two product $\mathcal{M}^{t} \mathcal{M}$ and $\mathcal{M} \mathcal{M}^{t}$, where " $t$ " is the transpose symbol. Last matrices are symmetric and hence they have eigen-decompositions: $\mathcal{M} \mathcal{M}^{t}=\mathbf{V} \Lambda \mathbf{V}^{+}, \quad \mathcal{M}^{t} \mathcal{M}=\mathbf{W} \Lambda \mathbf{W}^{+}$, where $\Lambda:=$ $\operatorname{diag}\left\{\lambda_{0}, \lambda_{1}, \ldots, \lambda_{N-1}\right\}$ and + denote the Hermitian conjugate. Then, it is well known that we can express $\mathcal{M}$ as the singular value decomposition $\mathcal{M}=\mathrm{VDW}^{+}$, where

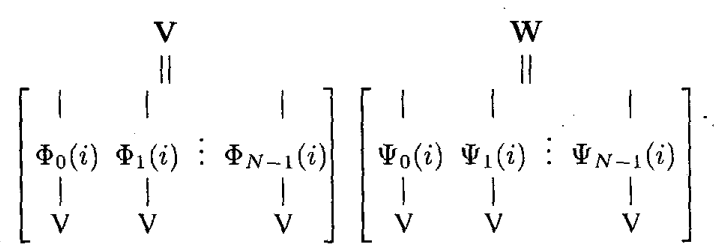

are matrices of eigen-vectors of $\mathcal{M} \mathcal{M}^{t}$ and $\mathcal{M}^{t} \mathcal{M}$ transforms, respectively, and $\mathbf{D}:=\sqrt{\Lambda}$. If $\alpha_{0}, \ldots, \alpha_{N-1}$ are an arbitrary real numbers then

$$
\mathcal{M}^{\alpha_{0}, \ldots, \alpha_{N-1}}:=\mathbf{V}\left[\mathbf{D}\left(\lambda_{0}^{\alpha_{0}}, \ldots, \lambda_{N-1}^{\alpha_{N-1}}\right)\right] \mathbf{W}^{+}
$$

is called the multi-parametric fractional $\mathcal{M}$-transform. If $\alpha_{i}=\alpha, \forall i=0,1, \ldots, N-1$ then this transform is called fractional $\mathcal{M}$-transform.

In 1937, Gondon wrote a paper called "Immersion of the Fourier transform in a continuous group of functional transformation" [2]. In 1961, Bargmann extended the fractional Fourier transform in his paper [1], in which he gave definition of the fractional Fourier transform, one based on Hermite polynomials as an integral transformation. If $H_{n}(\sqrt{2 \pi} t)$ is a Hermite polynomial of order $n$ then functions

$$
\Psi_{n}(t)=\frac{2^{1 / 4}}{\sqrt{2^{n} n !}} H_{n}(\sqrt{2 \pi} t) \exp \left(-\pi t^{2}\right)
$$

for $n=0,1,2, \ldots$ are eigen-functions of the Fourier transform

$$
\mathcal{F}\left[\Psi_{n}(t)\right]=\frac{1}{2 \pi} \int_{-\infty}^{+\infty} \Psi_{n}(t) e^{2 \pi j t \tau} d t=\lambda_{n} \Psi_{n}(t),
$$

with $\lambda_{n}=i^{n}$ being the eigen-value corresponding to the $n$th eigen-function. They form an orthogonal set of functions on the interval $(-\infty, \infty)$ with respect to weight function $e^{\pi t^{2}}$ :

$$
\begin{gathered}
\left\langle\Psi_{n}(t) \mid \Psi_{m}(t)\right\rangle=\int_{-\infty}^{+\infty} e^{\pi t^{2}} \Psi_{n}(t) \Psi_{m}(t) d t= \\
=\int_{-\infty}^{\infty} e^{-\pi t^{2}} \frac{2^{\frac{1}{4}}}{2^{n} n !} H_{n}(\sqrt{2 \pi} t) \frac{2^{\frac{1}{4}}}{2^{m} m !} H_{m}(\sqrt{2 \pi} t) d t=\delta_{m n} .
\end{gathered}
$$


According to Bargmann the fractional Fourier transform $\mathcal{F}^{\alpha}$ is defined through its the eigen-functions by

$$
\begin{gathered}
\mathcal{F}^{\alpha}=\left[\mathcal{F}^{\alpha}(\omega, t)\right]:=\left[\sum_{n=0}^{\infty} \lambda_{n}^{\alpha} \Psi_{n}(\omega) \Psi_{n}(t)\right]= \\
=\frac{\sqrt{2}}{2^{n} n !}\left[e^{\pi\left(\omega^{2}+t^{2}\right)} \sum_{n=0}^{\infty} \lambda_{n}^{\alpha} H_{n}(\sqrt{2 \pi} \omega) H_{n}(\sqrt{2 \pi} t)\right]= \\
=\frac{e^{\frac{j \pi}{4}\left(\alpha-\operatorname{sgn} \sin \frac{\pi \alpha}{2}\right)}}{\sqrt{\sin \frac{\pi \alpha}{2}}}\left[e^{j \pi \operatorname{ctg}\left(\frac{\pi \alpha}{2}\right)\left(\omega^{2}-2 \omega t \csc (\varphi)+t^{2}\right)}\right],
\end{gathered}
$$

where $\mathcal{F}^{\alpha}(\omega, t)$ is the kernel of the fractional Fourier transform. Obviously, a functions $\Psi_{n}(t)$ are eigen-functions of the fractional Fourier transform $\mathcal{F}^{\alpha}\left[\Psi_{n}(t)\right]=\lambda_{n}^{\alpha} \Psi_{n}(t)$ corresponding to the $n$th eigen-values $\lambda_{n}^{\alpha}, n=0,1,2, \ldots$ Of course for $\alpha=1 \mathcal{F}^{1}(\omega, t)=e^{j \omega t}$.

In 1980, Namias reinvented the fractional Fourier transform again in his paper [17]. This approach was extended by McBride and Kerr [16]. The fractional Fourier transform was restricted to pure mathematical purposes. Very few publications appeared. Then Mendlovic and Ozaktas introduced the fractional Fourier transform into the field of optics [18] in 1993: Afterwards, Lohmann [15] reinvented the fractional Fourier transform based on the Wigner-distribution function and opened the fractional Fourier transform to bulk-optics applications. In the series of papers [10],[19]-[22] authors developed the fast algorithms for a wide class of classical fractional transforms.

In this paper, we introduce the classical and quantum fractional Haar-Wavelet transforms and develop corresponding fast classical and quantum algorithms.

\section{Classical Haar-Wavelet transforms}

The Haar-Wavelet transform can be defined from the Haar functions and has the following factorization [9]:

$$
\mathbf{H W}_{2^{n}}=\prod_{i=1}^{n}\left[\left(\mathcal{F}_{2} \otimes I_{2^{n-i}}\right) \oplus I_{2^{n}-2^{n-i+1}}\right] \Pi_{2^{n}}
$$

where $\mathcal{F}_{2}=\frac{\sqrt{2}}{2}\left[\begin{array}{rr}1 & 1 \\ 1 & -1\end{array}\right]$ is the Walsh $(2 \times 2)-$ transform, where $\Pi_{2^{n}}$ is the perfect shuffle permutation matrix [5]. Classical description of $\Pi_{2^{n}}$ can be given by describing its effect on a given vector. If $\mathbf{v}=$ $\left(v_{0}, v_{1}, \ldots, v_{2^{n}-2}, v_{2^{n}-1}\right)$ is a $2^{n} \mathrm{D}$ vector, then the vector $\mathbf{w}=\Pi_{2^{n}} \mathbf{v}$ is obtained by splitting $\mathbf{v}$ in half and the shuffling the top and bottom halves of the deck. Alternatively, a description of the matrix $\Pi_{2^{n}}$, in terms of its elements $\Pi_{i j}$, for $i, j=0,1, \ldots, 2^{n}-1$, can be given as

$$
\Pi_{i j}= \begin{cases}1, & \text { if } j=i / 2 \text { and is even, or if } j=\frac{i-1}{2}+2^{n-1} \\ & \text { and } i \text { is odd, } \\ 0, & \text { otherwise. }\end{cases}
$$

The short description of $\Pi_{2^{n}}$ can be given by the left cyclic bit-shift of $i$-indexes $v_{\left(i_{n-1}, i_{n-2}, \ldots, i_{1}, i_{0}\right.}$ $\Pi_{2^{n}}\left(i_{n-1}, i_{n-2}, \ldots, i_{1}, i_{0}\right)=\left(i_{0}, i_{n-1}, i_{n-2}, \ldots, i_{1}\right)$. Note, that $\Pi_{2^{n}}^{t}$ performs the right cyclic bit-shift operation, i.e. $\Pi_{2^{n}}^{t}\left(i_{n-1}, i_{n-2}, \ldots, i_{1}, i_{0}\right)=\left(i_{n-2}, \ldots, i_{1}, i_{0}, i_{n-1}\right)$.

The perfect shuffle permutation matrix $\Pi_{2^{n}}$ has the following factorization [5]:

$$
\Pi_{2^{n}}=\prod_{i=2}^{n}\left(I_{2^{n-i}} \otimes \Pi_{4} \otimes I_{2^{i-2}}\right),
$$

where $\Pi_{4}$ is the "bit swap" operator, i.e., $\Pi_{4}\left(i_{1}, i_{0}\right):=$ $\left(i_{0}, i_{1}\right)$.

There are two families of generalized Haar transforms [7]-[14]. The first family (discrete controlled) has the following form:

$$
\begin{aligned}
& \quad \mathrm{HW}_{2^{n}}^{\left(k_{1}, k_{2}, \ldots, k_{n}\right)}:= \\
& =\prod_{i=1}^{n}\left[\left(I_{2^{k_{i}}} \otimes \mathcal{F}_{2} \otimes I_{2^{n-i}}\right) \oplus I_{2^{n}-2^{n-i+1-k_{i}}}\right] \Pi_{2^{n}},
\end{aligned}
$$

where the set of numbers $\left(k_{1}, k_{2}, \ldots, k_{n}\right)$ marks (and controls) the generalized Haar transforms, moreover, $0 \leq k_{1} \leq$ $0,0 \leq k_{2} \leq 1, \ldots, 0 \leq k_{n} \leq n-1$. In particular, $H_{2^{n}}^{(0,0, \ldots, 0)}=H_{2^{n}}$ is the standard Haar transform and $H_{2^{n}}^{(0,1, \ldots, n-1)}=W_{2^{n}}$ is the Walsh transform.

The second family (discrete and continuous controlled) contains the multi-parametric Haar-Wavelet transforms of the following form:

$$
\begin{gathered}
\mathbf{H W}_{\left(\varphi_{1}, \varphi_{2}, \ldots, \varphi_{n}\right)}^{\left(k_{1}, k_{2}, \ldots, k_{n}\right)}:= \\
=\prod_{i=1}^{n}\left[\left(I_{2^{k_{i}}} \otimes \mathbf{C S}_{2}\left(\varphi_{i}\right) \otimes I_{2^{n-i}}\right) \oplus I_{\left.2^{n}-2^{n-i+1-k_{i}}\right]}\right] \Pi_{2^{n}},(6) \\
\text { where } \mathbf{C S}_{2}\left(\varphi_{i}\right):=\left[\begin{array}{rr}
\cos \varphi & \sin \varphi \\
\sin \varphi & -\cos \varphi
\end{array}\right] . \text { Obviously, } \\
\mathbf{H W}_{\left(\frac{\pi}{4}, \frac{\pi}{4}, \ldots, \frac{\pi}{4}\right)}^{\left(k_{1}, k_{2}, \ldots, k_{n}\right)}=\mathbf{H W}_{2^{n}}^{\left(k_{1}, k_{2}, \ldots, k_{n}\right)} \text { and } \\
\mathbf{H W}_{\left(\frac{\pi}{4}, \frac{\pi}{4}, \ldots, \frac{\pi}{4}\right)}^{(0,1)}=\mathbf{H W}_{2^{n}} .
\end{gathered}
$$

For $\operatorname{CS}_{2}(\varphi)$ we have the following eigen-decomposition:

$$
\begin{gathered}
\operatorname{CS}_{2}(\varphi)=\left[\begin{array}{cc}
\cos \varphi & \sin \varphi \\
\sin \varphi & -\cos \varphi
\end{array}\right]= \\
{\left[\begin{array}{rr}
\cos \frac{\varphi}{2} & \sin \frac{\varphi}{2} \\
-\sin \frac{\varphi}{2} & \cos \frac{\varphi}{2}
\end{array}\right]\left[\begin{array}{rr}
1 & \\
& -1
\end{array}\right]\left[\begin{array}{cc}
\cos \frac{\varphi}{2} & -\sin \frac{\varphi}{2} \\
\sin \frac{\varphi}{2} & \cos \frac{\varphi}{2}
\end{array}\right]=}
\end{gathered}
$$




$$
=\operatorname{Rot}_{2}\left(\frac{\varphi}{2}\right) \mathbf{D}_{2}(1,-1) \overline{\boldsymbol{R o t}}_{2}\left(\frac{\varphi}{2}\right)
$$

where

$$
\begin{aligned}
& \operatorname{Rot}_{2}\left(\frac{\varphi}{2}\right)=\left[\begin{array}{rr}
\cos \frac{\varphi}{2} & \sin \frac{\varphi}{2} \\
-\sin \frac{\varphi}{2} & \cos \frac{\varphi}{2}
\end{array}\right], \mathbf{D}_{2}(1,-1)=\left[\begin{array}{ll}
1 & \\
& -1
\end{array}\right] . \\
& \text { In particular, } \mathcal{F}_{2}=\frac{\sqrt{2}}{2}\left[\begin{array}{rr}
1 & 1 \\
1 & -1
\end{array}\right]= \\
& =\left[\begin{array}{rr}
\cos \frac{\pi}{8} & \sin \frac{\pi}{8} \\
-\sin \frac{\pi}{8} & \cos \frac{\pi}{8}
\end{array}\right]\left[\begin{array}{rr}
1 & \\
& -1
\end{array}\right]\left[\begin{array}{ll}
\cos \frac{\pi}{8} & -\sin \frac{\pi}{8} \\
\sin \frac{\pi}{8} & \cos \frac{\pi}{8}
\end{array}\right]= \\
& =\operatorname{Rot}_{2}\left(\frac{\pi}{8}\right) \mathbf{D}_{2}(1,-1) \overline{\mathbf{R o t}}_{2}\left(\frac{\pi}{8}\right) .
\end{aligned}
$$

where $\cos \frac{\pi}{8}=\frac{\sqrt{2+\sqrt{2}}}{2}$ and $\sin \frac{\pi}{8}=\frac{\sqrt{2-\sqrt{2}}}{2}$.

$$
\text { Obviously, } \quad \operatorname{CS}_{2}^{\left(\alpha_{1}, \alpha_{2}\right)}(\varphi)=
$$$$
=\left[\begin{array}{cc}
\cos \frac{\varphi}{2} & \sin \frac{\varphi}{2} \\
-\sin \frac{\varphi}{2} & \cos \frac{\varphi}{2}
\end{array}\right]\left[\begin{array}{ll}
e^{2 \pi j \alpha_{1}} & \\
& e^{\pi j \alpha_{2}}
\end{array}\right]\left[\begin{array}{rr}
\cos \frac{\varphi}{2} & -\sin \frac{\varphi}{2} \\
\sin \frac{\varphi}{2} & \cos \frac{\varphi}{2}
\end{array}\right]=
$$

$$
=\boldsymbol{R o t}_{2}\left(\frac{-\varphi}{2}\right) \mathbf{D}_{2}\left(e^{2 \pi j \alpha_{1}}, e^{\pi j \alpha_{2}}\right) \operatorname{Rot}_{2}\left(\frac{\varphi}{2}\right),
$$

and

$$
\mathcal{F}_{2}^{\left(\alpha_{1}, \alpha_{2}\right)}=
$$

$$
\begin{gathered}
=\left[\begin{array}{rr}
\cos \frac{\pi}{8} & \sin \frac{\pi}{8} \\
-\sin \frac{\pi}{8} & \cos \frac{\pi}{8}
\end{array}\right] \cdot\left[\begin{array}{rr}
e^{2 \pi j \alpha_{1}} & \\
& e^{\pi j \alpha_{2}}
\end{array}\right]\left[\begin{array}{rr}
\cos \frac{\pi}{8} & -\sin \frac{\pi}{8} \\
\sin \frac{\pi}{8} & \cos \frac{\pi}{8}
\end{array}\right]= \\
=\boldsymbol{R o t}_{2}\left(\frac{\pi}{8}\right) \mathbf{D}_{2}\left(e^{2 \pi j \alpha_{1}}, e^{\pi j \alpha_{2}}\right) \overline{\boldsymbol{R o t}}_{2}\left(\frac{\pi}{8}\right)
\end{gathered}
$$

are the fractional $\mathbf{C S}_{2}$ and Walsh $(2 \times 2)$-transforms.

\section{Classical fractional Haar-Wavelet trans- forms}

The substitution of (9) into (6) gives the SVD of HaarWavelet transforms

$$
\begin{aligned}
& \mathbf{H W}_{\left(\varphi_{1}, \varphi_{2}, \ldots, \varphi_{n}\right)}^{\left(k_{1}, k_{2}, \ldots, k_{n}\right)}:= \\
& =\prod_{i=1}^{n}\left[\left(I_{2^{k_{i}}} \otimes \operatorname{Rot}_{2}\left(\frac{\varphi_{i}}{2}\right) \otimes I_{2^{n-i}}\right) \oplus I_{2^{n}-2^{n-i+1-k_{i}}}\right] \times \\
& \times \prod_{i=1}^{n}\left[\left(I_{2^{k_{i}}} \otimes \mathrm{D}_{2}(\overline{\mathrm{I}},-1) \otimes I_{2^{n-i}}\right) \oplus I_{2^{n}-2^{n-i+1-k_{i}}}\right] \times \\
& \times \prod_{i=1}^{n}\left[\left(I_{2^{k_{i}}} \otimes \overline{\mathbf{R o t}}_{2}\left(\frac{\varphi_{i}}{2}\right) \otimes I_{2^{n-i}}\right) \oplus I_{2^{n}-2^{n-i+1-k_{i}}}\right] \Pi_{2^{n}}=
\end{aligned}
$$

$$
\begin{aligned}
& =\left[\mathbf{V}_{\left(\varphi_{1}, \ldots, \varphi_{n}\right)}^{\left(k_{1}, \ldots, k_{n}\right)}\right] \mathbf{D}_{2^{n}}\left[\mathbf{W}_{\left(\varphi_{1}, \ldots, \psi_{n}\right)}^{\left(k_{1}, \ldots, k_{n}\right)}\right] \\
& \text { where } \quad \mathbf{V}_{\left(\varphi_{1}, \ldots, \varphi_{n}\right)}^{\left(k_{1}, \ldots, k_{n}\right)}:= \\
& =\prod_{i=1}^{n}\left[\left(I_{2^{k_{i}}} \otimes \operatorname{Rot}_{2}\left(\frac{\varphi_{i}}{2}\right) \otimes I_{2^{n-i}}\right) \oplus I_{2^{n}-2^{n-i+1-k_{i}}}\right] \Pi_{2^{n}} \\
& \mathrm{D}_{2^{n}}:=\prod_{i=1}^{n}\left[\left(I_{2^{k_{i}}} \otimes \mathrm{D}_{2}(1,-1) \otimes I_{2^{n-i}}\right) \oplus I_{2^{n}-2^{n-i+1-k_{i}}}\right], \\
& \mathbf{W}_{\left(\varphi_{1}, \ldots, \varphi_{n}\right)}^{\left(k_{1}, \ldots, k_{n}\right)}:= \\
& =\prod_{i=1}^{n}\left[\left(I_{2^{k_{i}}} \otimes \operatorname{Rot}_{2}\left(\frac{\varphi_{i}}{2}\right) \otimes I_{2^{n-i}}\right) \oplus I_{2^{n}-2^{n-i+1-k_{i}}}\right] \Pi_{2^{n}} .
\end{aligned}
$$

Now we can define two types of classical Haar-Wavelet transforms.

1. The $2 n$-parametric fractional Haar-Wavelet transforms with a separable diagonal matrix

$$
\begin{aligned}
& \mathbf{H W}_{\left(\varphi_{1}, \varphi_{2}, \ldots, \varphi_{n}\right)}^{\left(k_{1}, k_{2}, \ldots, k_{n}\right)}\left(\alpha_{1,1}, \alpha_{1,2}, \ldots, \alpha_{1, n}, \alpha_{2, n}\right):= \\
& =\prod_{i=1}^{n}\left[\left(I_{2^{k_{i}}} \otimes \operatorname{Rot}_{2}\left(\frac{\varphi_{i}}{2}\right) \otimes I_{2^{n-i}}\right) \oplus I_{2^{n}-2^{n-i+1-k_{i}}}\right] \times \\
& \times \prod_{i=1}^{n}\left[\left(I_{2^{k_{i}}} \otimes \mathbf{D}_{2}\left(e^{2 \pi j \alpha_{1, i}}, e^{\pi j \alpha_{2, i}}\right) \otimes I_{2^{n-i}}\right) \oplus I_{\left.2^{n}-2^{n-i+1-k_{i}}\right] \times}\right. \\
& \times \prod_{i=1}^{n}\left[\left(I_{2^{k_{i}}} \otimes \overline{\operatorname{Rot}}_{2}\left(\frac{\varphi_{i}}{2}\right) \otimes I_{2^{n-i}}\right) \oplus \bar{I}_{2^{n}-2^{n-i+1-k_{i}}}\right] \Pi_{2^{n}}:= \\
& =\prod_{i=1}^{n}\left[\left(I_{2^{k_{i}}} \otimes \mathbf{C S}_{2}^{\left(\alpha_{1, i}, \alpha_{2, i}\right)}\left(\varphi_{i}\right) \otimes I_{2^{n-i}}\right) \oplus I_{2^{n}-2^{n-i+1-k_{i}}}\right] \Pi_{2^{n}}
\end{aligned}
$$

In particular,

$$
=\mathbf{H} \mathbf{W}_{2^{n}}^{\left(k_{1}, k_{2}, \ldots, k_{n}\right)}\left(\alpha_{1,1}, \alpha_{1,2}, \ldots, \alpha_{1, n}, \alpha_{2, n}\right)=
$$

$=\prod_{i=1}^{n}\left[\left(I_{2^{k_{i}}} \otimes \mathbf{C S}_{2}^{\left(\alpha_{1, i}, \alpha_{2, i}\right)}\left(\frac{\pi}{8}\right) \otimes I_{2^{n-i}}\right) \oplus I_{2^{n}-2^{n-i+1-k_{i}}}\right] \Pi_{2^{n}}$.

and

$$
\begin{gathered}
\mathbf{H W}_{2^{n}}\left(\alpha_{1,1}, \alpha_{1,2}, \ldots, \alpha_{1, n}, \alpha_{2, n}\right)= \\
=\prod_{i=1}^{n}\left[\mathbf{C S}_{2}^{\left(\alpha_{1, i}, \alpha_{2, i}\right)}\left(\frac{\pi}{8}\right) \otimes I_{2^{n-i}} \oplus I_{2^{n}-2^{n-i+1}}\right] \Pi_{2^{n}}
\end{gathered}
$$

are classical fractional Haar-Wavelet transform associated with $\mathbf{H W}_{2^{n}}^{\left(k_{1}, k_{2}, \ldots, k_{n}\right)}$ and $\mathbf{H} \mathbf{W}_{2^{n}}$, respectively. We see that the fractional Haar-Wavelet transforms (15)-(17) have the Haar-like fast algorithms. 
2. The $2^{n}$-parametric fractional Walsh transform with a non-separable diagonal matrix

$$
\begin{gathered}
\mathbf{H W}_{\left(\mathbf{W}_{1}, \varphi_{2}, \ldots, \varphi_{n}\right)}^{\left(k_{1}, k_{2}, \ldots, k_{n}\right)}\left(\alpha_{0}, \alpha_{1}, \ldots, \alpha_{2^{n}-1}\right):= \\
=\prod_{i=1}^{n}\left[\left(I_{2^{k_{i}}} \otimes \operatorname{Rot}_{2}\left(\frac{\varphi}{2}\right) \otimes I_{2^{n-i}}\right) \oplus I_{\left.2^{n}-2^{n-i+1-k_{i}}\right] \times}\right. \\
\times\left[\mathbf{D}_{2^{n}}\left(\alpha_{0}, \alpha_{1}, \ldots, \alpha_{2^{n}-1}\right)\right] \times \\
\times \prod_{i=1}^{n}\left[\left(I_{2^{k_{i}}} \otimes \overline{\mathbf{R o t}}_{2}\left(\frac{\varphi}{2}\right) \otimes I_{2^{n-i}}\right) \oplus I_{2^{n}-2^{n-i+1-k_{i}}}\right] \Pi_{2^{n}}
\end{gathered}
$$

where $\mathbf{D}_{2^{n}}\left(\alpha_{0}, \alpha_{1}, \ldots, \alpha_{2^{n}-1}\right)$ is a diagonal $\left(2^{n} \times 2^{n}\right)$ matrix.

\section{Quantum fractional Haar-Wavelet trans- form}

All operations in quantum computation are realized by means of transformations on the QU-BIT's contained in a quantum register. The possible transformations a quantum computer can carry out are the elements of unitary group $\mathcal{U}\left(\mathbf{C}^{2^{n}}\right)$. A quantum logic gate is an elementary quantum computing device which performs a fixed unitary transformation on selected QU-BIT's in a fixed period of time. A transformation gate takes an input quantum state and produces a modified output quantum state. The gates have the same number of inputs as outputs, and a gate of $n$ inputs carries a unitary transformation of the group $\mathcal{U}\left(\mathbf{C}^{2^{n}}\right)$, i.e., a generalized rotation in the Hilbert space $\mathbf{C}^{2^{n}}$. To study the complexity of performing unitary transformations on QU$2^{n}$ REG, we introduce two types of quantum logic gates [4][6],[23]:

- Local unitary operations on $k-$ th QU-BIT are matrices of the form $U_{2^{n}}^{(k)}:=I_{2^{k-1}} \otimes U_{2} \otimes I_{2^{n-k}}$, where $U_{2}$ is an element of the unitary group $\mathcal{U}\left(\mathrm{C}^{2}\right)$ of $(2 \times 2)$-matrices. For these operations we have

$$
\begin{gathered}
{\left[I_{2^{i-1}} \otimes U_{2} \otimes I_{2^{n-i}}\right]\left|q_{1}\right\rangle \otimes \ldots \otimes\left|q_{i}\right\rangle \otimes \ldots \otimes\left|q_{n}\right\rangle=} \\
\left.=\left|q_{1}\right\rangle \otimes \ldots \otimes\left[U_{2}\left|q_{i}\right\rangle\right] \otimes|\ldots \otimes| q_{n}\right\rangle .
\end{gathered}
$$

- For any unitary $\left[2^{n-k} \times 2^{n-k}\right]$-transformation $U_{2^{n-k}}$ we define the $n$-BIT transformation $U_{2^{n}}^{\overline{1, k}}$ by

$$
U_{2^{n}}^{\overline{I_{k}}}:=I_{2^{n}-2^{n-k}} \oplus U_{2^{n-k}} .
$$

This operator is called the $(\overline{1, k})$-controlled $U_{2^{n}}$-operator, where $U_{2^{n}}^{\overline{1, k}}$ acts as identity transforms in the subspace $\mathrm{C}^{2^{n}-2^{n-k}}$ and as $U_{2^{n-k}}$ in the second subspace $\mathrm{C}^{2^{k}}$, if $q_{1}, q_{2}, \ldots, q_{k}=1$ and $q_{k+1}, \ldots, q_{n} \neq 0$. Here $C^{2^{n}}=$ $\mathbf{C}^{2^{n}-2^{n-k}} \oplus \mathbf{C}^{2^{k}}$. In particular, if $U_{2^{n-k}}$ is the tensor product of $n-k(2 \times 2)$-matrices $U_{2^{n-k}}:=U_{2, k+1} \otimes U_{2, k+2} \otimes$ $\cdots \otimes U_{2, n}$, then

$$
\begin{gathered}
{\left[U_{2, k+1} \otimes \cdots \otimes U_{2, n}\right]_{2^{n}}^{\overline{1, k}} \times} \\
\times\left[\left|q_{1}\right\rangle \otimes \ldots \otimes\left|q_{k}\right\rangle \otimes\left|q_{k+1}\right\rangle \otimes \ldots \otimes\left|q_{n}\right\rangle\right]= \\
\left|q_{1}\right\rangle \otimes \ldots \otimes\left|q_{k}\right\rangle \otimes\left[U_{2, k+1}^{\overline{1, k}}\left|q_{k+1}\right\rangle\right] \otimes \ldots \otimes\left[U_{2, n}^{\overline{1, k}}\left|q_{n}\right\rangle\right] \cdot(21) \\
\text { If } U_{2^{n-k}}:=U_{2^{n-k}}^{(j)}=I_{2^{j-1}} \otimes U_{2} \otimes I_{2^{n-k-j}}, \text { then } \\
{\left[I_{2^{j-1}} \otimes U_{2} \otimes I_{2^{n-k-j}}\right]_{2^{n}}^{\overline{1, k}}\left[\left|q_{1}\right\rangle \otimes \ldots \otimes\left|q_{k+j}\right\rangle \otimes \ldots \otimes\left|q_{n}\right\rangle\right]=} \\
=\left|q_{1}\right\rangle \otimes \ldots \otimes\left[U_{2}^{\overline{1, k}}\left|q_{k+j}\right\rangle\right] \otimes \ldots \otimes\left|q_{n}\right\rangle .
\end{gathered}
$$

- For any diagonal unitary $(2 \times 2)$-transformation $\mathbf{D}_{2}^{\left(t_{1}, \ldots, t_{n-1}, 0\right)}$ we define the $\left(2^{n} \times 2^{n}\right)$-transformation by

$$
\begin{aligned}
& \mathbf{D}_{2^{n}}^{\left(t_{1}, \ldots, t_{n-1}, 0\right)}=\mathbf{D}_{2^{n}}\left(e^{2 j \pi \alpha_{\left(t_{1}, \ldots, t_{n-1}, 0\right)}} e^{\left.j \pi \alpha_{\left(t_{1}, \ldots, t_{n-1}, 1\right)}\right)}=\right. \\
& =I_{\left(t_{1}, \ldots, t_{n-1}, 0\right)} \oplus \mathbf{D}_{2}^{\left(t_{1}, \ldots, t_{n-1}, 0\right)} \oplus I_{\left(\bar{t}_{1}, \ldots, \bar{t}_{n-1}, 0\right)}
\end{aligned}
$$

This operator is called the $\left(t_{1}, \ldots, t_{n-1}, 0\right)$-controlled operator. Obviously,

$$
\begin{gathered}
\mathcal{Q} \mathcal{D}_{2^{n}}^{\left(t_{1}, \ldots, t_{n-1}, 0\right)}= \\
=\prod_{t_{1}=0}^{1} \ldots \prod_{t_{n-1}=0}^{1}\left[I_{\left(t_{1}, \ldots, t_{n-1}, 0\right)} \oplus \mathbf{D}_{2^{n}}^{\left(t_{1}, \ldots, t_{n-1}, 0\right)} \oplus I_{\left(\bar{t}_{1}, \ldots, \bar{t}_{n 1-1}, 0\right)}\right]
\end{gathered}
$$

We shall use a standard graphical notation for quantum circuits. [4]-[6],[23] In this notation the tensor structure of the Hilbert space $\mathbf{C}^{2^{n}}=\mathbf{C}^{2} \otimes \mathbf{C}^{2} \otimes \ldots \otimes \mathbf{C}^{2}$ is reflected by drawing $n$ parallel lines (=quantum wires) each of which represents one tensor component $\mathbf{C}^{2}$. A box sitting just on one wire denotes a local transformation $U_{2^{n}}^{(i)}$ whereas the

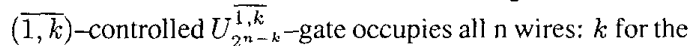
control and $n-k$ for the transformation (see Fig. 1).

The quantum network (gate array)is a quantum computing device consisting of quantum logic gates whose computational steps are synchronised in time. The quantum network is the natural quantum generalization of the acyclic combinatorial logic circuits studied in conventional computational complexity theory. The output of some of the gates are connected by wires to the input of others and they interconnected without fanout or feedback by quantum wires. A quantum computer will be viewed here as a quantum network (or a family of quantum networks). Quantum computation is defined as unitary evolution of the network which takes its initial state "input" into some final state "output".

In order to realize quantum fast fractional Haar-Wavelet transforms, we introduce 
- input "time" quantum register

QU-2 ${ }^{n} \operatorname{REG}(|\mathbf{t}\rangle):=$\begin{tabular}{|l|l|l|l|}
$\left|t_{1}\right\rangle$ & $\left|t_{2}\right\rangle$ & $\cdots$ & $\left|t_{n}\right\rangle$ \\
\hline
\end{tabular}

- output "frequency" quantum register

$$
\mathrm{QU}-2^{n} \operatorname{REG}(|\omega\rangle):=\begin{array}{|l|l|l|l|}
\left|\omega_{1}\right\rangle & \left|\omega_{2}\right\rangle & \cdots & \left|\omega_{n}\right\rangle \\
\hline
\end{array}
$$

According to (19), (20) we can introduce quantum counterparts of transforms (12), (14), (15) and (18)

$$
\begin{gathered}
\mathcal{Q H} \mathcal{W}_{2^{n}}^{1}=\prod_{i=1}^{n}\left[\operatorname{CS}_{2}^{\alpha_{1, i}, \alpha_{2, i}}\right]_{2^{n}}^{\overline{(i+1, n)}} \\
\mathcal{Q} \mathcal{V}_{2^{n}}=\prod_{i=1}^{n}\left[\operatorname{Rot}_{2}\left(\frac{\varphi}{2}\right)\right]^{\overline{(i+1, n)}} \\
\mathcal{Q} \mathcal{W}_{2^{n}}=\prod_{i=1}^{n}\left[\overline{\operatorname{Rot}}_{2}\left(\frac{\varphi}{2}\right)\right]^{\overline{(i+1, n)}} \Pi_{2^{n}} \\
\mathcal{Q H} \mathcal{W}_{2^{n}}^{2}=\left[\mathcal{Q} \mathcal{V}_{2^{n}}\right]\left[\mathcal{Q D} \mathcal{D}_{2^{n}}^{\left(t_{1}, \ldots, t_{n-1}, 0\right)}\right]\left[\mathcal{Q} \mathcal{W}_{2^{n}}\right]
\end{gathered}
$$

In the language of quantum circuits, these transforms are presented in Fig. 2 and Fig. 3, respectively.

\section{Acknowledgement}

This research was performed at the Signal Processing Laboratory, Tampere University of Technology, Finland. The work was also supported by Ural State Techical University, Ekaterinburg, Russia.

\section{References}

[1] V. Bargmann. On a Hilbert space of analytic functions and an associated integral transform. Part 1. Commun. Pure Appl. Math., 14:187-214, 1961.

[2] E. U. Condon. Immersion of the Fourier transform in a continuous group of functional transforms. Proc. Nat. Acad. Sci., USA, 12:158-164, 1937.

[3] R. Creutzburg, E. Rundblad, and V. Labunets. Fast algorithms for fractional Fourier transforms. Proc: of IEEEEURASIP Workshop on Nonlinear Signal and Image Processing, Antalyá, Turkey, pages 383-387, June 1999.

[4] A. Fijany and C. P. Williams. Quantum wavelet transforms: Fast algorithms and complete circuits. LANL preprint quant-ph/9800904, September 1998.

[5] P. Hoyer. Efficient quantum transforms. LANL preprint quant-ph/9702028, February 1997.

[6] A. Y. Kitaev. Quantum measurement and the Abelian Stabilizer problem. LANL preprint quant-ph/9702028, February 1997.

[7] G. S. Kolmogorov and V. G. Labunets. Strategy of of fast multiparapeters transforms (in Russian). Orthogonal Methods for the Application in Signal Processing and System. Analysis, Urals Polytechnical Institute Press, Sverdlovsk, Russia, pages 4-24, 1983.
[8] G. S. Kolmogorov and V. G. Labunets. Fast algorithms in approximate bases of Krawtschick polynomials (in Russian). Fast Digital Systems of Information Processing, Republic seminar, Uzgorod, Russia, pages 17-20, 1984.

[9] V. G. Labinets. Generalized Haar transforms (in Russian). In: Multivalued elements, structures, systems. Institute of Cybernetics of Ukraian Academy of Sciences Press: Kiev, pages 46-58, 1983.

[10] E. Labunets and V. Labunets. Fast fractional Fourier transforms. Proc. of Eusipco-98, Rhodes, Greece, pages 17571760, September 1998.

[11] V. G. Labunets. New unitary transforms with fast algorithm structures (in Russian). Experimental investigations automatization. Institute of Technical Cybernetics of Belorussian Academy of Sciences Press, Minsk, Belorussia, pages 61$69,1982$.

[12] V. G. Labunets. Unified approach to fast algorithms of unitary transforms (in Russian). Multivalued elements, structures, systems, Institute of Cybernetics of Ukraian Academy of Sciences, Kiev, pages 46-58, 1983.

[13] V. G. Labunets. Fast multiparameters transforms (in Russian). Proceedings of Radioelectronics, 8:89-109, 1985.

[14] V. G. Labunets. Fast nonlinear multiparameters transforms (in Russian). In: Methods and microelectronical devices of information numerical transform and processing, All-Union Scientific Conference, Moscow, pages 64-74, 1987.

[15] A. W. Lohmann. Image rotation, Wigner rotation, and the fractional order Fourier transform. J. Opt. Soc. Am. A., 10:2181-2186, 1993.

[16] A. C. McBride and F. H. Kerr. On Namias' fractional Fourier transforms. IMA J. Appl. Math., 39:131-265, 1987.

[17] V. Namias. The fractional order Fourier transform and its application to quatum mechanics. J. Inst. Math. Appl., 25:131265,1980 .

[18] H. M. Ozaktas and D. Mendlovic. Fourier transform of fractional order and their optical interpretation. Opt. Commun., 110:163-169, 1993.

[19] E. Rundblad, V. Labunets, J. Astola, K. Egiazarian, and S. Polovnev. Fast fractional unitary transforms. Proc. of Conf. Computer Science and Information Technologies, Yerevan, Armenia, pages 223-226, 1999.

[20] E. Rundblad, V. Labunets, J. Astola, K. Egiazarian, and A. Smaga. Fast fractional Fourier and Hartley transforms. Proc. of the 1999 Finnish Signal Processing Symposium, Oulu, Finland, pages 291-297, 1999.

[21] E. Rundblad-Labunets, V. Labunets, J. Astola, and K. Egiazarian. Fast fractional Fourier-Clifford transforms. Second International Workshop on Transforms and Filter Banks, Tampere, Finland, TICSP Series, 5:376-405, March 1999.

[22] A. Smaga, E. Labunets, and V. Labunets. New fast algorithms for fractional Fourier transforms. Automatic and $1 n$ formation Technologies. "UGTU-UPI Scientific Schools"Report N.5, pages 265-272, 1999.

[23] V. V. Vedral, A. Barenco, and A. Ekert. Quantum networks for elememtary arithmetic operations. Physical Review A, 54:147-153, 1996. 


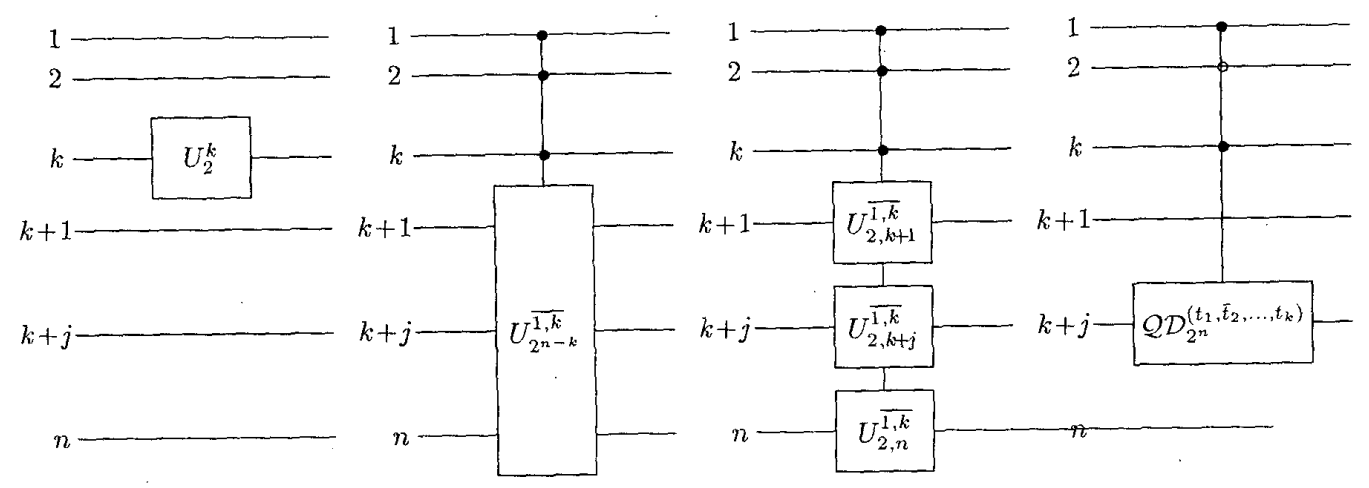

Figure 1. Quantum gates for a) $U_{2^{n}}^{(k)}$, b) $(\overline{1, k})$-controlled $U_{2^{n-k}}^{\overline{1, k}}$-operator, c) $\left[U_{2, k+1}^{\overline{1, k}} \otimes \cdots \otimes U_{2, n}^{\overline{1, k}}\right]$ and d) $Q \mathcal{D}_{2^{n}}^{\left(t_{1}, \bar{t}_{2}, \ldots, t_{k}\right)}$, respectively

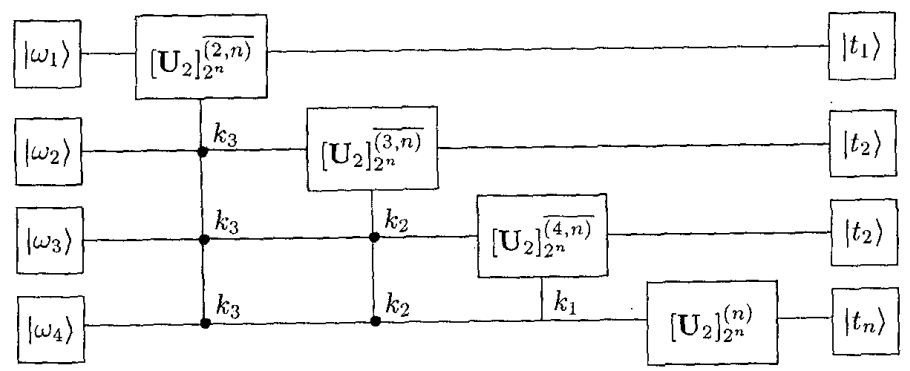

Figure 3. Quantum fast $2^{n}$-parametric Haar-Wavelet transform $\mathcal{Q H} \mathcal{W}_{2^{n}}^{1}$, if $\left[\mathbf{U}_{2}\right]_{2^{n}}^{\overline{(2, n)}}=\left[\mathbf{C S}_{2}^{\alpha_{1, i}, \alpha_{2, i}}\right]_{2^{n}}^{\overline{(i+1, n)}}$ or left $\mathcal{Q V}_{2^{n}}$ (right $\mathcal{Q W} 2_{2^{n}}$ ) eigen-transforms, if $\left[\mathbf{U}_{2}\right]_{2^{n}}^{(2, n)}=\left[\operatorname{Rot}_{2}\left(\frac{\varphi}{2}\right)\right]^{(i+k, n)}$, for $n=4$

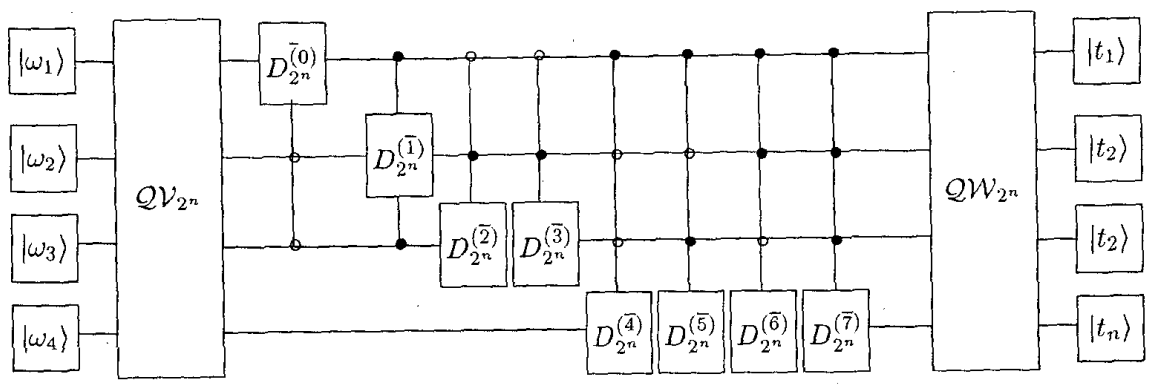

Figure 3. Quantum fast $2^{n}$-parametric Haar-Wavelet transform $\mathcal{Q H} \mathcal{W}_{2^{n}}^{\left(\alpha_{0}, \alpha_{1}, \ldots, \alpha_{2^{n}-1}\right)}, n=4$ 